\title{
Research Article \\ Extension of Jensen's Inequality for Operators without Operator Convexity
}

\author{
Jadranka Mićić, ${ }^{1}$ Zlatko Pavić, $_{,}{ }^{2}$ and Josip Pečarić ${ }^{3}$ \\ ${ }^{1}$ Faculty of Mechanical Engineering and Naval Architecture, University of Zagreb, Ivana Lučića 5, \\ 10000 Zagreb, Croatia \\ ${ }^{2}$ Mechanical Engineering Faculty, University of Osijek, Trg Ivane Brlić Mažuranić 2, \\ 35000 Slavonski Brod, Croatia \\ ${ }^{3}$ Faculty of Textile Technology, University of Zagreb, Prilaz baruna Filipovića 30, 10000 Zagreb, Croatia \\ Correspondence should be addressed to Jadranka Mićić, jmicic@fsb.hr
}

Received 7 March 2011; Accepted 13 April 2011

Academic Editor: Nikolaos Papageorgiou

Copyright @ 2011 Jadranka Mićić et al. This is an open access article distributed under the Creative Commons Attribution License, which permits unrestricted use, distribution, and reproduction in any medium, provided the original work is properly cited.

We give an extension of Jensen's inequality for $n$-tuples of self-adjoint operators, unital $n$-tuples of positive linear mappings, and real-valued continuous convex functions with conditions on the operators' bounds. We also study operator quasiarithmetic means under the same conditions.

\section{Introduction}

We recall some notations and definitions. Let $\mathbb{B}(H)$ be the $\mathrm{C}^{*}$-algebra of all bounded linear operators on a Hilbert space $H$ and $1_{H}$ stands for the identity operator. We define bounds of a self-adjoint operator $A \in B(H)$ :

$$
m_{A}=\inf _{\|x\|=1}\langle A x, x\rangle, \quad M_{A}=\sup _{\|x\|=1}\langle A x, x\rangle
$$

for $x \in H$. If $\operatorname{Sp}(A)$ denotes the spectrum of $A$, then $\operatorname{Sp}(A)$ is real and $\operatorname{Sp}(A) \subseteq\left[m_{A}, M_{A}\right]$.

Mond and Pečarić in [1] proved the following version of Jensen's operator inequality

$$
f\left(\sum_{i=1}^{n} w_{i} \Phi_{i}\left(A_{i}\right)\right) \leq \sum_{i=1}^{n} w_{i} \Phi_{i}\left(f\left(A_{i}\right)\right),
$$

for operator convex functions $f$ defined on an interval $I$, where $\Phi_{i}: \mathcal{B}(H) \rightarrow \mathcal{B}(K), i=$ $1, \ldots, n$, are unital positive linear mappings, $A_{1}, \ldots, A_{n}$ are self-adjoint operators with the spectra in $I$, and $w_{1}, \ldots, w_{n}$ are nonnegative real numbers with $\sum_{i=1}^{n} w_{i}=1$. 
Hansen et al. gave in [2] a generalization of (1.2) for a unital field of positive linear mappings. Recently, Mićić et al. in [3] gave a generalization of this results for a not-unital field of positive linear mappings.

Very recently, Mićić et al. gave in [4, Theorem 1] a version of Jensen's operator inequality without operator convexity as follows.

Theorem A. Let $\left(A_{1}, \ldots, A_{n}\right)$ be an n-tuple of self-adjoint operators $A_{i} \in \mathbb{B}(H)$ with bounds $m_{i}$ and $M_{i}, m_{i} \leq M_{i}, i=1, \ldots, n$. Let $\left(\Phi_{1}, \ldots, \Phi_{n}\right)$ be an $n$-tuple of positive linear mappings $\Phi_{i}: \mathbb{B}(H) \rightarrow$ $\mathbb{B}(K), i=1, \ldots, n$, such that $\sum_{i=1}^{n} \Phi_{i}\left(1_{H}\right)=1_{K}$. If

$$
\left(m_{C}, M_{C}\right) \cap\left[m_{i}, M_{i}\right]=\emptyset \text { for } i=1, \ldots, n,
$$

where $m_{C}$ and $M_{C}, m_{C} \leq M_{C}$, are bounds of the self-adjoint operator $C=\sum_{i=1}^{n} \Phi_{i}\left(A_{i}\right)$, then

$$
f\left(\sum_{i=1}^{n} \Phi_{i}\left(A_{i}\right)\right) \leq \sum_{i=1}^{n} \Phi_{i}\left(f\left(A_{i}\right)\right)
$$

holds for every continuous convex function $f: I \rightarrow \mathbb{R}$ provided that the interval I contains all $m_{i}, M_{i}$.

If $f: I \rightarrow \mathbb{R}$ is concave, then the reverse inequality is valid in (1.4).

In the same paper [4], we study the quasiarithmetic operator mean:

$$
\mathcal{M}_{\varphi}(\mathbf{A}, \mathbf{\Phi}, n)=\varphi^{-1}\left(\sum_{i=1}^{n} \Phi_{i}\left(\varphi\left(A_{i}\right)\right)\right),
$$

where $\left(A_{1}, \ldots, A_{n}\right)$ is an $n$-tuple of self-adjoint operators in $B(H)$ with the spectra in $I$, $\left(\Phi_{1}, \ldots, \Phi_{n}\right)$ is an $n$-tuple of positive linear mappings $\Phi_{i}: \mathcal{B}(H) \rightarrow \mathcal{B}(K)$ such that $\sum_{i=1}^{n} \Phi_{i}\left(1_{H}\right)=1_{K}$, and $\varphi: I \rightarrow \mathbb{R}$ is a continuous strictly monotone function.

The following results about the monotonicity of this mean are proven in $[4$, Theorem 3].

Theorem B. Let $\left(A_{1}, \ldots, A_{n}\right)$ and $\left(\Phi_{1}, \ldots, \Phi_{n}\right)$ be as in the definition of the quasiarithmetic mean (1.5). Let $m_{i}$ and $M_{i}, m_{i} \leq M_{i}$, be the bounds of $A_{i}, i=1, \ldots, n$. Let $\varphi, \psi: I \rightarrow \mathbb{R}$ be continuous strictly monotone functions on an interval I which contains all $m_{i}, M_{i}$. Let $m_{\varphi}$ and $M_{\varphi}, m_{\varphi} \leq M_{\varphi}$, be the bounds of the mean $\boldsymbol{M}_{\varphi}(\mathbf{A}, \mathbf{\Phi}, n)$, such that

$$
\left(m_{\varphi}, M_{\varphi}\right) \cap\left[m_{i}, M_{i}\right]=\emptyset, \quad \text { for } i=1, \ldots, n .
$$

If one of the following conditions

(i) $\psi \circ \varphi^{-1}$ is convex and $\psi^{-1}$ is operator monotone,

(i') $\psi \circ \varphi^{-1}$ is concave and $-\psi^{-1}$ is operator monotone,

is satisfied, then

$$
\mathcal{M}_{\varphi}(\mathrm{A}, \boldsymbol{\Phi}, n) \leq \mathcal{M}_{\psi}(\mathbf{A}, \boldsymbol{\Phi}, n) .
$$


If one of the following conditions

(ii) $\psi \circ \varphi^{-1}$ is concave and $\psi^{-1}$ is operator monotone,

(ii') $\psi \circ \varphi^{-1}$ is convex and $-\psi^{-1}$ is operator monotone,

is satisfied, then the reverse inequality is valid in (1.7).

In this paper we study an extension of Jensen's inequality given in Theorem A. As an application of this result, we give an extension of Theorem B for a version of the quasiarithmetic mean (1.5) with an $n$-tuple of positive linear mappings which is not unital.

\section{Extension of Jensens Operator Inequality}

In Theorem A we prove that Jensen's operator inequality holds for every continuous convex function and for every $n$-tuple of self-adjoint operators $\left(A_{1}, \ldots, A_{n}\right)$, for every $n$-tuple of positive linear mappings $\left(\Phi_{1}, \ldots, \Phi_{n}\right)$ in the case when the interval with bounds of the operator $A=\sum_{i=1}^{n} \Phi_{i}\left(A_{i}\right)$ has no intersection points with the interval with bounds of the operator $A_{i}$ for each $i=1, \ldots, n$, that is, when

$$
\left(m_{A}, M_{A}\right) \cap\left[m_{i}, M_{i}\right]=\emptyset, \quad \text { for } i=1, \ldots, n,
$$

where $m_{A}$ and $M_{A}, m_{A} \leq M_{A}$, are the bounds of $A$, and $m_{i}$ and $M_{i}, m_{i} \leq M_{i}$, are the bounds of $A_{i}, i=1, \ldots, n$.

It is interesting to consider the case when $\left(m_{A}, M_{A}\right) \cap\left[m_{i}, M_{i}\right]=\emptyset$ is valid for several $i \in\{1, \ldots, n\}$, but not for all $i=1, \ldots, n$. We study it in the following theorem.

Theorem 2.1. Let $\left(A_{1}, \ldots, A_{n}\right)$ be an $n$-tuple of self-adjoint operators $A_{i} \in B(H)$ with the bounds $m_{i}$ and $M_{i}, m_{i} \leq M_{i}, i=1, \ldots, n$. Let $\left(\Phi_{1}, \ldots, \Phi_{n}\right)$ be an $n$-tuple of positive linear mappings $\Phi_{i}$ : $B(H) \rightarrow B(K)$, such that $\sum_{i=1}^{n} \Phi_{i}\left(1_{H}\right)=1_{K}$. For $1 \leq n_{1}<n$, one denotes $m=\min \left\{m_{1}, \ldots, m_{n_{1}}\right\}$, $M=\max \left\{M_{1}, \ldots, M_{n_{1}}\right\}$, and $\sum_{i=1}^{n_{1}} \Phi_{i}\left(1_{H}\right)=\alpha 1_{K}, \sum_{i=n_{1}+1}^{n} \Phi_{i}\left(1_{H}\right)=\beta 1_{K}$, where $\alpha, \beta>0$, $\alpha+\beta=1$. If

$$
(m, M) \cap\left[m_{i}, M_{i}\right]=\emptyset, \quad \text { for } i=n_{1}+1, \ldots, n,
$$

and one of two equalities

$$
\frac{1}{\alpha} \sum_{i=1}^{n_{1}} \Phi_{i}\left(A_{i}\right)=\frac{1}{\beta} \sum_{i=n_{1}+1}^{n} \Phi_{i}\left(A_{i}\right)=\sum_{i=1}^{n} \Phi_{i}\left(A_{i}\right)
$$

is valid, then

$$
\frac{1}{\alpha} \sum_{i=1}^{n_{1}} \Phi_{i}\left(f\left(A_{i}\right)\right) \leq \sum_{i=1}^{n} \Phi_{i}\left(f\left(A_{i}\right)\right) \leq \frac{1}{\beta} \sum_{i=n_{1}+1}^{n} \Phi_{i}\left(f\left(A_{i}\right)\right)
$$

holds for every continuous convex function $f: I \rightarrow \mathbb{R}$ provided that the interval I contains all $m_{i}, M_{i}, i=1, \ldots, n$.

If $f: I \rightarrow \mathbb{R}$ is concave, then the reverse inequality is valid in (2.4). 
Proof. We prove only the case when $f$ is a convex function.

Let us denote

$$
A=\frac{1}{\alpha} \sum_{i=1}^{n_{1}} \Phi_{i}\left(A_{i}\right), \quad B=\frac{1}{\beta} \sum_{i=n_{1}+1}^{n} \Phi_{i}\left(A_{i}\right), \quad C=\sum_{i=1}^{n} \Phi_{i}\left(A_{i}\right)
$$

It is easy to verify that $A=B$ or $B=C$ or $A=C$ implies $A=B=C$.

(a) Let $m<M$. Since $f$ is convex on $[m, M]$ and $\left[m_{i}, M_{i}\right] \subseteq[m, M]$ for $i=1, \ldots, n_{1}$, then

$$
f(t) \leq \frac{M-t}{M-m} f(m)+\frac{t-m}{M-m} f(M), \quad t \in\left[m_{i}, M_{i}\right] \text { for } i=1, \ldots, n_{1},
$$

but since $f$ is convex on all $\left[m_{i}, M_{i}\right]$ and $(m, M) \cap\left[m_{i}, M_{i}\right]=\emptyset$ for $i=n_{1}+1, \ldots, n$, then

$$
f(t) \geq \frac{M-t}{M-m} f(m)+\frac{t-m}{M-m} f(M), \quad t \in\left[m_{i}, M_{i}\right] \text { for } i=n_{1}+1, \ldots, n .
$$

Since $m_{i} 1_{H} \leq A_{i} \leq M_{i} 1_{H}, i=1, \ldots, n_{1}$, it follows from (2.6) that

$$
f\left(A_{i}\right) \leq \frac{M 1_{H}-A_{i}}{M-m} f(m)+\frac{A_{i}-m 1_{H}}{M-m} f(M), \quad i=1, \ldots, n_{1} .
$$

Applying a positive linear mapping $\Phi_{i}$ and summing, we obtain

$$
\sum_{i=1}^{n_{1}} \Phi_{i}\left(f\left(A_{i}\right)\right) \leq \frac{M \alpha 1_{K}-\sum_{i=1}^{n_{1}} \Phi_{i}\left(A_{i}\right)}{M-m} f(m)+\frac{\sum_{i=1}^{n_{1}} \Phi_{i}\left(A_{i}\right)-m \alpha 1_{K}}{M-m} f(M),
$$

since $\sum_{i=1}^{n_{1}} \Phi_{i}\left(1_{H}\right)=\alpha 1_{K}$. It follows that

$$
\frac{1}{\alpha} \sum_{i=1}^{n_{1}} \Phi_{i}\left(f\left(A_{i}\right)\right) \leq \frac{M 1_{K}-A}{M-m} f(m)+\frac{A-m 1_{K}}{M-m} f(M)
$$

Similarly to (2.10) in the case $m_{i} 1_{H} \leq A_{i} \leq M_{i} 1_{H}, i=n_{1}+1, \ldots, n$, it follows from (2.7)

$$
\frac{1}{\beta} \sum_{i=n_{1}+1}^{n} \Phi_{i}\left(f\left(A_{i}\right)\right) \geq \frac{M 1_{K}-B}{M-m} f(m)+\frac{B-m 1_{K}}{M-m} f(M) .
$$

Combining (2.10) and (2.11) and taking into account that $A=B$, we obtain

$$
\frac{1}{\alpha} \sum_{i=1}^{n_{1}} \Phi_{i}\left(f\left(A_{i}\right)\right) \leq \frac{1}{\beta} \sum_{i=n_{1}+1}^{n} \Phi_{i}\left(f\left(A_{i}\right)\right)
$$


It follows that

$$
\begin{aligned}
\frac{1}{\alpha} \sum_{i=1}^{n_{1}} \Phi_{i}\left(f\left(A_{i}\right)\right) & =\sum_{i=1}^{n_{1}} \Phi_{i}\left(f\left(A_{i}\right)\right)+\frac{\beta}{\alpha} \sum_{i=1}^{n_{1}} \Phi_{i}\left(f\left(A_{i}\right)\right) \quad(\text { by } \alpha+\beta=1) \\
& \leq \sum_{i=1}^{n_{1}} \Phi_{i}\left(f\left(A_{i}\right)\right)+\sum_{i=n_{1}+1}^{n} \Phi_{i}\left(f\left(A_{i}\right)\right) \quad(\text { by }(2.12)) \\
& =\sum_{i=1}^{n} \Phi_{i}\left(f\left(A_{i}\right)\right) \\
& =\sum_{i=1}^{n_{1}} \Phi_{i}\left(f\left(A_{i}\right)\right)+\sum_{i=n_{1}+1}^{n} \Phi_{i}\left(f\left(A_{i}\right)\right) \\
& \left.\leq \frac{\alpha}{\beta} \sum_{i=n_{1}+1}^{n} \Phi_{i}\left(f\left(A_{i}\right)\right)+\sum_{i=n_{1}+1}^{n} \Phi_{i}\left(f\left(A_{i}\right)\right) \quad \text { (by }(2.12)\right) \\
& =\frac{1}{\beta} \sum_{i=n_{1}+1}^{n} \Phi_{i}\left(f\left(A_{i}\right)\right) \quad(\text { by } \alpha+\beta=1),
\end{aligned}
$$

which gives the desired double inequality (2.4).

(b) Let $m=M$. Since $\left[m_{i}, M_{i}\right] \subseteq[m, M]$ for $i=1, \ldots, n_{1}$, then $A_{i}=m 1_{H}$ and $f\left(A_{i}\right)=$ $f(m) 1_{H}$ for $i=1, \ldots, n_{1}$. It follows that

$$
\frac{1}{\alpha} \sum_{i=1}^{n_{1}} \Phi_{i}\left(A_{i}\right)=m 1_{K}, \quad \frac{1}{\alpha} \sum_{i=1}^{n_{1}} \Phi_{i}\left(f\left(A_{i}\right)\right)=f(m) 1_{K} .
$$

On the other hand, since $f$ is convex on $I$, we have

$$
f(t) \geq f(m)+l(m)(t-m) \quad \text { for every } t \in I,
$$

where $l$ is the subdifferential of $f$. Replacing $t$ by $A_{i}$ for $i=n_{1}+1, \ldots, n$, applying $\Phi_{i}$ and summing, we obtain from (2.15) and (2.14) that

$$
\begin{aligned}
\frac{1}{\beta} \sum_{i=n_{1}+1}^{n} \Phi_{i}\left(f\left(A_{i}\right)\right) & \geq f(m) 1_{K}+l(m)\left(\frac{1}{\beta} \sum_{i=n_{1}+1}^{n} \Phi_{i}\left(A_{i}\right)-m 1_{K}\right) \\
& =f(m) 1_{K}=\frac{1}{\alpha} \sum_{i=1}^{n_{1}} \Phi_{i}\left(f\left(A_{i}\right)\right) .
\end{aligned}
$$

So (2.12) holds again. The remaining part of the proof is the same as in the case (a). follows.

As a special case of Theorem 2.1 we can obtain Theorem A. We give this proof as Another Proof of Theorem A. Let the assumptions of Theorem A be valid. We prove only the case when $f$ is a convex function. 
We define operators $B_{i} \in B(H), i=1, \ldots, n+1$, by $B_{1}=C=\sum_{i=1}^{n} \Phi_{i}\left(A_{i}\right)$ and $B_{i}=A_{i-1}$, $i=2, \ldots, n+1$. Then $m_{B_{1}}=m_{C}$, and $M_{B_{1}}=M_{C}$ are the bounds of $B_{1}$ and $m_{B_{i}}=m_{i-1}$, and $M_{B_{i}}=M_{i-1}$ are the ones of $B_{i}, i=2, \ldots, n+1$. We have

$$
\left(m_{B_{1}}, M_{B_{1}}\right) \cap\left[m_{B_{i}}, M_{B_{i}}\right]=\emptyset \quad \text { for } i=2, \ldots, n+1,
$$

since (1.3) holds. Also, we define mappings $\Psi_{i}: B(H) \rightarrow B(K)$ by $\Psi_{1}(B)=(1 / 2) B$ and $\Psi_{i}(B)=(1 / 2) \Phi_{i-1}(B), i=2, \ldots, n+1$. Then we have $\sum_{i=1}^{n+1} \Psi_{i}\left(1_{H}\right)=1_{K}$ and

$$
\sum_{i=1}^{n+1} \Psi_{i}\left(B_{i}\right)=\Psi_{1}\left(B_{1}\right)+\sum_{i=2}^{n+1} \Psi_{i}\left(B_{i}\right)=\frac{1}{2} \sum_{i=1}^{n} \Phi_{i}\left(A_{i}\right)+\frac{1}{2} \sum_{i=1}^{n} \Phi_{i}\left(A_{i}\right)=B_{1}
$$

It follows that

$$
2 \Psi_{1}\left(B_{1}\right)=\sum_{i=1}^{n+1} \Psi_{i}\left(B_{i}\right)=2 \sum_{i=2}^{n+1} \Psi_{i}\left(B_{i}\right)
$$

Taking into account (2.17) and (2.19), we can apply Theorem 2.1 for $n_{1}=1$ and $B_{i}, \Psi_{i}$ as above. We get

$$
2 \Psi_{1}\left(f\left(B_{1}\right)\right) \leq \sum_{i=1}^{n+1} \Psi_{i}\left(f\left(B_{i}\right)\right) \leq 2 \sum_{i=2}^{n+1} \Psi_{i}\left(f\left(B_{i}\right)\right)
$$

that is,

$$
f\left(\sum_{i=1}^{n} \Phi_{i}\left(A_{i}\right)\right) \leq \frac{1}{2} f\left(\sum_{i=1}^{n} \Phi_{i}\left(A_{i}\right)\right)+\frac{1}{2} \sum_{i=1}^{n} \Phi_{i}\left(f\left(A_{i}\right)\right) \leq \sum_{i=1}^{n} \Phi_{i}\left(f\left(A_{i}\right)\right),
$$

which gives the desired inequality (1.4).

Remark 2.2. We obtain the equivalent inequality to the one in Theorem 2.1 in the case when $\sum_{i=1}^{n} \Phi_{i}\left(1_{H}\right)=\gamma 1_{K}$, for some positive scalar $\gamma$. If $\alpha+\beta=\gamma$ and one of two equalities

$$
\frac{1}{\alpha} \sum_{i=1}^{n_{1}} \Phi_{i}\left(A_{i}\right)=\frac{1}{\beta} \sum_{i=n_{1}+1}^{n} \Phi_{i}\left(A_{i}\right)=\frac{1}{\gamma} \sum_{i=1}^{n} \Phi_{i}\left(A_{i}\right)
$$

is valid, then

$$
\frac{1}{\alpha} \sum_{i=1}^{n_{1}} \Phi_{i}\left(f\left(A_{i}\right)\right) \leq \frac{1}{\gamma} \sum_{i=1}^{n} \Phi_{i}\left(f\left(A_{i}\right)\right) \leq \frac{1}{\beta} \sum_{i=n_{1}+1}^{n} \Phi_{i}\left(f\left(A_{i}\right)\right)
$$

holds for every continuous convex function $f$. 
Remark 2.3. Let the assumptions of Theorem 2.1 be valid.

(1) We observe that the following inequality

$$
f\left(\frac{1}{\beta} \sum_{i=n_{1}+1}^{n} \Phi_{i}\left(A_{i}\right)\right) \leq \frac{1}{\beta} \sum_{i=n_{1}+1}^{n} \Phi_{i}\left(f\left(A_{i}\right)\right),
$$

holds for every continuous convex function $f: I \rightarrow \mathbb{R}$.

Indeed, by the assumptions of Theorem 2.1 we have

$$
m \alpha 1_{H} \leq \sum_{i=1}^{n_{1}} \Phi_{i}\left(f\left(A_{i}\right)\right) \leq M \alpha 1_{H}, \quad \frac{1}{\alpha} \sum_{i=1}^{n_{1}} \Phi_{i}\left(A_{i}\right)=\frac{1}{\beta} \sum_{i=n_{1}+1}^{n} \Phi_{i}\left(A_{i}\right),
$$

which implies that

$$
m 1_{H} \leq \sum_{i=n_{1}+1}^{n} \frac{1}{\beta} \Phi_{i}\left(f\left(A_{i}\right)\right) \leq M 1_{H}
$$

Also $(m, M) \cap\left[m_{i}, M_{i}\right]=\emptyset$ for $i=n_{1}+1, \ldots, n$ and $\sum_{i=n_{1}+1}^{n}(1 / \beta) \Phi_{i}\left(1_{H}\right)=1_{K}$ hold. So we can apply Theorem A on operators $A_{n_{1}+1}, \ldots, A_{n}$ and mappings $(1 / \beta) \Phi_{i}$ and obtain the desired inequality.

(2) We denote by $m_{C}$ and $M_{C}$ the bounds of $C=\sum_{i=1}^{n} \Phi_{i}\left(A_{i}\right)$. If $\left(m_{C}, M_{C}\right) \cap\left[m_{i}, M_{i}\right]=$ $\emptyset, i=1, \ldots, n_{1}$ or $f$ is an operator convex function on $[m, M]$, then the double inequality (2.4) can be extended from the left side if we use Jensen's operator inequality (see [3, Theorem 2.1]):

$$
\begin{aligned}
f\left(\sum_{i=1}^{n} \Phi_{i}\left(A_{i}\right)\right) & =f\left(\frac{1}{\alpha} \sum_{i=1}^{n_{1}} \Phi_{i}\left(A_{i}\right)\right) \\
& \leq \frac{1}{\alpha} \sum_{i=1}^{n_{1}} \Phi_{i}\left(f\left(A_{i}\right)\right) \leq \sum_{i=1}^{n} \Phi_{i}\left(f\left(A_{i}\right)\right) \\
& \leq \frac{1}{\beta} \sum_{i=n_{1}+1}^{n} \Phi_{i}\left(f\left(A_{i}\right)\right) .
\end{aligned}
$$

Example 2.4. If neither assumptions that $\left(m_{C}, M_{C}\right) \cap\left[m_{i}, M_{i}\right]=\emptyset, i=1, \ldots, n_{1}$ nor $f$ is operator convex in Remark 2.3(2). is satisfied and if $1<n_{1}<n$, then (2.4) cannot be extended by Jensen's operator inequality, since it is not valid. Indeed, for $n_{1}=2$ we define mappings $\Phi_{1}, \Phi_{2}: M_{3}(\mathbb{C}) \rightarrow M_{2}(\mathbb{C})$ by $\Phi_{1}\left(\left(a_{i j}\right)_{1 \leq i, j \leq 3}\right)=(\alpha / 2)\left(a_{i j}\right)_{1 \leq i, j \leq 2}, \Phi_{2}=\Phi_{1}$. Then $\Phi_{1}\left(I_{3}\right)+$ $\Phi_{2}\left(I_{3}\right)=\alpha I_{2}$. If

$$
A_{1}=2\left(\begin{array}{lll}
1 & 0 & 1 \\
0 & 0 & 1 \\
1 & 1 & 1
\end{array}\right), \quad A_{2}=2\left(\begin{array}{lll}
1 & 0 & 0 \\
0 & 0 & 0 \\
0 & 0 & 0
\end{array}\right)
$$


then

$$
\left(\frac{1}{\alpha} \Phi_{1}\left(A_{1}\right)+\frac{1}{\alpha} \Phi_{2}\left(A_{2}\right)\right)^{4}=\frac{1}{\alpha^{4}}\left(\begin{array}{cc}
16 & 0 \\
0 & 0
\end{array}\right) \not \leq \frac{1}{\alpha}\left(\begin{array}{ll}
80 & 40 \\
40 & 24
\end{array}\right)=\frac{1}{\alpha} \Phi_{1}\left(A_{1}^{4}\right)+\frac{1}{\alpha} \Phi_{2}\left(A_{2}^{4}\right)
$$

for every $\alpha \in(0,1)$. We observe that $f(t)=t^{4}$ is not operator convex and $\left(m_{C}, M_{C}\right) \cap$

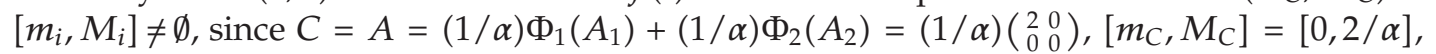
$\left[m_{1}, M_{1}\right] \subset[-1.60388,4.49396]$, and $\left[m_{2}, M_{2}\right]=[0,2]$.

With respect to Remark 2.2, we obtain the following obvious corollary of Theorem 2.1.

Corollary 2.5. Let $\left(A_{1}, \ldots, A_{n}\right)$ be an n-tuple of self-adjoint operators $A_{i} \in B(H)$ with the bounds $m_{i}$ and $M_{i}, m_{i} \leq M_{i}, i=1, \ldots, n$. For some $1 \leq n_{1}<n$, one denotes $m=\min \left\{m_{1}, \ldots, m_{n_{1}}\right\}$, $M=\max \left\{M_{1}, \ldots, M_{n_{1}}\right\}$. Let $\left(p_{1}, \ldots, p_{n}\right)$ be an $n$-tuple of nonnegative numbers, such that $0<$ $\sum_{i=1}^{n_{1}} p_{i}=\mathbf{p}_{\mathbf{n}_{1}}<\mathbf{p}_{\mathbf{n}}=\sum_{i=1}^{n} p_{i}$. If

$$
(m, M) \cap\left[m_{i}, M_{i}\right]=\emptyset \quad \text { for } i=n_{1}+1, \ldots, n,
$$

and one of two equalities

$$
\frac{1}{\mathbf{p}_{\mathbf{n}_{1}}} \sum_{i=1}^{n_{1}} p_{i} A_{i}=\frac{1}{\mathbf{p}_{\mathbf{n}}} \sum_{i=1}^{n} p_{i} A_{i}=\frac{1}{\mathbf{p}_{\mathbf{n}}-\mathbf{p}_{\mathbf{n}_{1}}} \sum_{i=n_{1}+1}^{n} p_{i} A_{i}
$$

is valid, then

$$
\frac{1}{\mathbf{p}_{\mathbf{n}_{1}}} \sum_{i=1}^{n_{1}} p_{i} f\left(A_{i}\right) \leq \frac{1}{\mathbf{p}_{\mathbf{n}}} \sum_{i=1}^{n} p_{i} f\left(A_{i}\right) \leq \frac{1}{\mathbf{p}_{\mathbf{n}}-\mathbf{p}_{\mathbf{n}_{1}}} \sum_{i=n_{1}+1}^{n} p_{i} f\left(A_{i}\right)
$$

holds for every continuous convex function $f: I \rightarrow \mathbb{R}$ provided that the interval I contains all $m_{i}, M_{i}, i=1, \ldots, n$.

If $f: I \rightarrow \mathbb{R}$ is concave, then the reverse inequality is valid in (2.32).

\section{Quasiarithmetic Means}

In this section we study an application of Theorem 2.1 to the quasiarithmetic mean with weight. For a subset $\left\{A_{p_{1}}, \ldots, A_{p_{2}}\right\}$ of $\left\{A_{1}, \ldots, A_{n}\right\}$ one denotes the quasiarithmetic mean by

$$
\mathcal{M}_{\varphi}\left(\gamma, \mathbf{A}, \boldsymbol{\Phi}, p_{1}, p_{2}\right)=\varphi^{-1}\left(\frac{1}{r} \sum_{i=p_{1}}^{p_{2}} \Phi_{i}\left(\varphi\left(A_{i}\right)\right)\right)
$$

where $\left(A_{p_{1}}, \ldots, A_{p_{2}}\right)$ are self-adjoint operators in $B(H)$ with the spectra in $I,\left(\Phi_{p_{1}}, \ldots, \Phi_{p_{2}}\right)$ are positive linear mappings $\Phi_{i}: \mathbb{B}(H) \rightarrow \mathbb{B}(K)$ such that $\sum_{i=p_{1}}^{p_{2}} \Phi_{i}\left(1_{H}\right)=\gamma 1_{K}$, and $\varphi: I \rightarrow \mathbb{R}$ is a continuous strictly monotone function.

The following theorem is an extension of Theorem B. 
Theorem 3.1. Let $\left(A_{1}, \ldots, A_{n}\right)$ be an $n$-tuple of self-adjoint operators in $\mathbb{B}(H)$ with the spectra in $I$, and let $\left(\Phi_{1}, \ldots, \Phi_{n}\right)$ be an $n$-tuple of positive linear mappings $\Phi_{i}: \mathcal{B}(H) \rightarrow \mathcal{B}(K)$ such that $\sum_{i=1}^{n} \Phi_{i}\left(1_{H}\right)=1_{K}$. Let $m_{i}$ and $M_{i}, m_{i} \leq M_{i}$ be the bounds of $A_{i}, i=1, \ldots, n$. Let $\varphi, \psi: I \rightarrow$ $\mathbb{R}$ be continuous strictly monotone functions on an interval $I$ which contains all $m_{i}, M_{i}$. For $1 \leq$ $n_{1}<n$, one denotes $m=\min \left\{m_{1}, \ldots, m_{n_{1}}\right\}, M=\max \left\{M_{1}, \ldots, M_{n_{1}}\right\}$, and $\sum_{i=1}^{n_{1}} \Phi_{i}\left(1_{H}\right)=\alpha 1_{K}$, $\sum_{i=n_{1}+1}^{n} \Phi_{i}\left(1_{H}\right)=\beta 1_{K}$, where $\alpha, \beta>0, \alpha+\beta=1$. Let

$$
(m, M) \cap\left[m_{i}, M_{i}\right]=\emptyset, \quad \text { for } i=n_{1}+1, \ldots, n,
$$

and let one of two equalities

$$
\mathcal{M}_{\varphi}\left(\alpha, \mathbf{A}, \mathbf{\Phi}, 1, n_{1}\right)=\mathcal{M}_{\varphi}(1, \mathbf{A}, \mathbf{\Phi}, 1, n)=\mathcal{M}_{\varphi}\left(\beta, \mathbf{A}, \mathbf{\Phi}, n_{1}+1, n\right)
$$

be valid.

If one of the following conditions

(i) $\psi \circ \varphi^{-1}$ is convex, and $\psi^{-1}$ is operator monotone,

(i') $\psi \circ \varphi^{-1}$ is concave and $-\psi^{-1}$ is operator monotone,

is satisfied, then

$$
\mathcal{M}_{\psi}\left(\alpha, \mathbf{A}, \mathbf{\Phi}, 1, n_{1}\right) \leq \mathcal{M}_{\psi}(1, \mathbf{A}, \boldsymbol{\Phi}, 1, n) \leq \mathcal{M}_{\psi}\left(\beta, \mathbf{A}, \boldsymbol{\Phi}, n_{1}+1, n\right) .
$$

If one of the following conditions

(i) $\psi \circ \varphi^{-1}$ is concave and $\psi^{-1}$ is operator monotone,

(ii') $\psi \circ \varphi^{-1}$ is convex and $-\psi^{-1}$ is operator monotone,

is satisfied, then the reverse inequality is valid in (3.4).

Proof. We only prove the case (i). Suppose that $\varphi$ is a strictly increasing function. Since $m 1_{H} \leq$ $A_{i} \leq M 1_{H}, i=1, \ldots, n_{1}$, implies $\varphi(m) 1_{K} \leq \varphi\left(A_{i}\right) \leq \varphi(M) 1_{K}$, then

$$
(m, M) \cap\left[m_{i}, M_{i}\right]=\emptyset \quad \text { for } i=n_{1}+1, \ldots, n
$$

implies

$$
(\varphi(m), \varphi(M)) \cap\left[\varphi\left(m_{i}\right), \varphi\left(M_{i}\right)\right]=\emptyset, \quad \text { for } i=n_{1}+1, \ldots, n
$$

Also, by using (3.3), we have

$$
\frac{1}{\alpha} \sum_{i=1}^{n_{1}} \Phi_{i}\left(\varphi\left(A_{i}\right)\right)=\sum_{i=1}^{n} \Phi_{i}\left(\varphi\left(A_{i}\right)\right)=\frac{1}{\beta} \sum_{i=n_{1}+1}^{n} \Phi_{i}\left(\varphi\left(A_{i}\right)\right) .
$$


Taking into account (3.6) and the above double equality, we obtain by Theorem 2.1

$$
\frac{1}{\alpha} \sum_{i=1}^{n_{1}} \Phi_{i}\left(f\left(\varphi\left(A_{i}\right)\right)\right) \leq \sum_{i=1}^{n} \Phi_{i}\left(f\left(\varphi\left(A_{i}\right)\right)\right) \leq \frac{1}{\beta} \sum_{i=n_{1}+1}^{n} \Phi_{i}\left(f\left(\varphi\left(A_{i}\right)\right)\right)
$$

for every continuous convex function $f: J \rightarrow \mathbb{R}$ on an interval $J$ which contains all $\left[\varphi\left(m_{i}\right), \varphi\left(M_{i}\right)\right]=\varphi\left(\left[m_{i}, M_{i}\right]\right), i=1, \ldots, n$.

Also, if $\varphi$ is strictly decreasing, then we check that (3.8) holds for convex $f: J \rightarrow \mathbb{R}$ on $J$ which contains all $\left[\varphi\left(M_{i}\right), \varphi\left(m_{i}\right)\right]=\varphi\left(\left[m_{i}, M_{i}\right]\right)$.

Putting $f=\psi \circ \varphi^{-1}$ in (3.8), we obtain

$$
\frac{1}{\alpha} \sum_{i=1}^{n_{1}} \Phi_{i}\left(\psi\left(A_{i}\right)\right) \leq \sum_{i=1}^{n} \Phi_{i}\left(\psi\left(A_{i}\right)\right) \leq \frac{1}{\beta} \sum_{i=n_{1}+1}^{n} \Phi_{i}\left(\psi\left(A_{i}\right)\right)
$$

Applying an operator monotone function $\psi^{-1}$ on the above double inequality, we obtain the desired inequality (3.4).

As a special case of Theorem 3.1 we can obtain Theorem B as follows.

Another Proof of Theorem B. We give the short version of the proof, since it is essentially the same as the one of Theorem A in Section 2.

Let the assumptions of Theorem B be valid, $\psi \circ \varphi^{-1}$ is convex and $\psi^{-1}$ is operator monotone.

Let $B_{1}=\varphi^{-1}\left(\sum_{i=1}^{n} \Phi_{i}\left(\varphi\left(A_{i}\right)\right)\right)$ and $B_{i}=A_{i-1}, i=2, \ldots, n+1$. Then $m_{B_{1}}=m_{\varphi}$, and $M_{B_{1}}=$ $M_{\varphi}$ are the bounds of $B_{1}$, and $m_{B_{i}}=m_{i-1}$, and $M_{B_{i}}=M_{i-1}$, are the ones of $B_{i}, i=2, \ldots, n+1$. We have

$$
\left(m_{B_{1}}, M_{B_{1}}\right) \cap\left[m_{B_{i}}, M_{B_{i}}\right]=\emptyset \text { for } i=2, \ldots, n+1,
$$

since (1.6) holds. Also, we define mappings $\Theta_{1}(B)=(1 / 2) B$ and $\Theta_{i}(B)=(1 / 2) \Phi_{i-1}(B), i=$ $2, \ldots, n+1$. Then we have $\sum_{i=1}^{n+1} \Theta_{i}\left(1_{H}\right)=1_{K}$ and

$$
\sum_{i=1}^{n+1} \Theta_{i}\left(\varphi\left(B_{i}\right)\right)=\frac{1}{2} \sum_{i=1}^{n} \Phi_{i}\left(\varphi\left(A_{i}\right)\right)+\frac{1}{2} \sum_{i=1}^{n} \Phi_{i}\left(\varphi\left(A_{i}\right)\right)=\varphi\left(B_{1}\right) .
$$

It follows that

$$
B_{1}=\mathcal{M}_{\varphi}\left(\frac{1}{2}, \mathbf{B}, \boldsymbol{\Theta}, 1,1\right)=\mathcal{M}_{\varphi}(1, \mathbf{B}, \boldsymbol{\Theta}, 1, n+1)=\mathcal{M}_{\varphi}\left(\frac{1}{2}, \mathbf{B}, \boldsymbol{\Theta}, 2, n+1\right) .
$$

So the assumptions of Theorem 3.1 are valid and it follows that

$$
B_{1}=\mathcal{M}_{\psi}\left(\frac{1}{2}, \mathbf{B}, \mathbf{\Theta}, 1,1\right) \leq \mathcal{M}_{\psi}(1, \mathbf{B}, \mathbf{\Theta}, 1, n+1) \leq \mathcal{M}_{\psi}\left(\frac{1}{2}, \mathbf{B}, \mathbf{\Theta}, 2, n+1\right)
$$


holds. Therefore, it follows that

$$
\varphi^{-1}\left(\sum_{i=1}^{n} \Phi_{i}\left(\varphi\left(A_{i}\right)\right)\right)=B_{1} \leq \psi^{-1}\left(\sum_{i=1}^{n} \Phi_{i}\left(\psi\left(A_{i}\right)\right)\right)
$$

which is the desired inequality (1.7).

In the remaining cases the proof is essentially the same as in the above cases.

Remark 3.2. Let the assumptions of Theorem 3.1 be valid.

(1) We observe that if one of the following conditions

(i) $\psi \circ \varphi^{-1}$ is convex and $\psi^{-1}$ is operator monotone,

(i') $\psi \circ \varphi^{-1}$ is concave and $-\psi^{-1}$ is operator monotone,

is satisfied, then the following obvious inequality (see Remark 2.3(1))

$$
\mathcal{M}_{\varphi}\left(\beta, \mathbf{A}, \boldsymbol{\Phi}, n_{1}+1, n\right) \leq \mathcal{M}_{\psi}\left(\beta, \mathbf{A}, \boldsymbol{\Phi}, n_{1}+1, n\right)
$$

holds.

(2) We denote by $m_{\varphi}$ and $M_{\varphi}$ the bounds of $\mathcal{M}_{\varphi}(1, \mathbf{A}, \mathbf{\Phi}, 1, n)$. If $\left(m_{\varphi}, M_{\varphi}\right) \cap\left[m_{i}, M_{i}\right]=$ $\emptyset, i=1, \ldots, n_{1}$, and one of two following conditions

(i) $\psi \circ \varphi^{-1}$ is convex and $\psi^{-1}$ is operator monotone

(ii) $\psi \circ \varphi^{-1}$ is concave and $-\psi^{-1}$ is operator monotone

is satisfied, or if one of the following conditions

(i') $\psi \circ \varphi^{-1}$ is operator convex and $\psi^{-1}$ is operator monotone,

(ii') $\psi \circ \varphi^{-1}$ is operator concave and $-\psi^{-1}$ is operator monotone,

is satisfied (see [4, Theorem B]), then the double inequality (3.4) can be extended from the left side as follows:

$$
\begin{aligned}
\mathcal{M}_{\varphi}(1, \mathbf{A}, \mathbf{\Phi}, 1, n) & =\mathcal{M}_{\varphi}\left(1, \mathbf{A}, \mathbf{\Phi}, 1, n_{1}\right) \\
& \leq \mathcal{M}_{\psi}\left(\alpha, \mathbf{A}, \mathbf{\Phi}, 1, n_{1}\right) \leq \mathcal{M}_{\psi}(1, \mathbf{A}, \mathbf{\Phi}, 1, n) \\
& \leq \mathcal{M}_{\psi}\left(\beta, \mathbf{A}, \mathbf{\Phi}, n_{1}+1, n\right) .
\end{aligned}
$$

(3) If neither assumptions that $\left(m_{\psi}, M_{\psi}\right) \cap\left[m_{i}, M_{i}\right]=\emptyset, i=1, \ldots, n_{1}$ nor $\psi \circ \varphi^{-1}$ is operator convex (or operator concave) is satisfied and if $1<n_{1}<n$, then (3.4) cannot be extended from the left side by $\mathcal{M}_{\varphi}\left(1, \mathbf{A}, \boldsymbol{\Phi}, 1, n_{1}\right)$ as above. It is easy to check it with a counterexample similarly to [4, Example 2].

We now give some particular results of interest that can be derived from Theorem 3.1. 
Corollary 3.3. Let $\left(A_{1}, \ldots, A_{n}\right)$ and $\left(\Phi_{1}, \ldots, \Phi_{n}\right), m_{i}, M_{i}, m, M, \alpha$, and $\beta$ be as in Theorem 3.1. Let $I$ be an interval which contains all $m_{i}, M_{i}$ and

$$
(m, M) \cap\left[m_{i}, M_{i}\right]=\emptyset, \quad \text { for } i=n_{1}+1, \ldots, n .
$$

If one of two equalities

$$
\mathcal{M}_{\varphi}\left(\alpha, \mathbf{A}, \mathbf{\Phi}, 1, n_{1}\right)=\mathcal{M}_{\varphi}(1, \mathbf{A}, \mathbf{\Phi}, 1, n)=\mathcal{M}_{\varphi}\left(\beta, \mathbf{A}, \mathbf{\Phi}, n_{1}+1, n\right)
$$

is valid, then

$$
\frac{1}{\alpha} \sum_{i=1}^{n_{1}} \Phi_{i}\left(A_{i}\right) \leq \sum_{i=1}^{n} \Phi_{i}\left(A_{i}\right) \leq \frac{1}{\beta} \sum_{i=n_{1}+1}^{n} \Phi_{i}\left(A_{i}\right)
$$

holds for every continuous strictly monotone function $\varphi: I \rightarrow \mathbb{R}$ such that $\varphi^{-1}$ is convex on $I$. But, if $\varphi^{-1}$ is concave, then the reverse inequality is valid in (3.19).

On the other hand, if one of two equalities

$$
\frac{1}{\alpha} \sum_{i=1}^{n_{1}} \Phi_{i}\left(A_{i}\right)=\sum_{i=1}^{n} \Phi_{i}\left(A_{i}\right)=\frac{1}{\beta} \sum_{i=n_{1}+1}^{n} \Phi_{i}\left(A_{i}\right)
$$

is valid, then

$$
\mathcal{M}_{\varphi}\left(\alpha, \mathbf{A}, \mathbf{\Phi}, 1, n_{1}\right) \leq \mathcal{M}_{\varphi}(1, \mathbf{A}, \mathbf{\Phi}, 1, n) \leq \mathcal{M}_{\varphi}\left(\beta, \mathbf{A}, \mathbf{\Phi}, n_{1}+1, n\right)
$$

holds for every continuous strictly monotone function $\varphi: I \rightarrow \mathbb{R}$ such that one of the following conditions

(i) $\varphi$ is convex and $\varphi^{-1}$ is operator monotone,

(i') $\varphi$ is concave and $-\varphi^{-1}$ is operator monotone,

is satisfied. But, if one of the following conditions

(ii) $\varphi$ is concave and $\varphi^{-1}$ is operator monotone,

(ii') $\varphi$ is convex and $-\varphi^{-1}$ is operator monotone,

is satisfied, then the reverse inequality is valid in (3.21).

Proof. The proof of (3.19) follows from Theorem 3.1 by replacing $\psi$ with the identity function, while the proof of (3.21) follows from the same theorem by replacing $\varphi$ with the identity function and $\psi$ with $\varphi$. 
As a special case of the quasiarithmetic mean (3.1) we can study the weighted power mean as follows. For a subset $\left\{A_{p_{1}}, \ldots, A_{p_{2}}\right\}$ of $\left\{A_{1}, \ldots, A_{n}\right\}$ one denotes this mean by

$$
M^{[r]}\left(\gamma, \mathbf{A}, \boldsymbol{\Phi}, p_{1}, p_{2}\right)= \begin{cases}\left(\frac{1}{r} \sum_{i=p_{1}}^{p_{2}} \Phi_{i}\left(A_{i}^{r}\right)\right)^{1 / r}, & r \in \mathbb{R} \backslash\{0\}, \\ \exp \left(\frac{1}{r} \sum_{i=p_{1}}^{p_{2}} \Phi_{i}\left(\ln \left(A_{i}\right)\right)\right), & r=0,\end{cases}
$$

where $\left(A_{p_{1}}, \ldots, A_{p_{2}}\right)$ are strictly positive operators, and $\left(\Phi_{p_{1}}, \ldots, \Phi_{p_{2}}\right)$ are positive linear mappings $\Phi_{i}: \mathcal{B}(H) \rightarrow \mathcal{B}(K)$ such that $\sum_{i=p_{1}}^{p_{2}} \Phi_{i}\left(1_{H}\right)=\gamma 1_{K}$.

We obtain the following corollary by applying Theorem 3.1 to the above mean.

Corollary 3.4. Let $\left(A_{1}, \ldots, A_{n}\right)$ be an $n$-tuple of strictly positive operators in $\boldsymbol{B}(H)$ and let $\left(\Phi_{1}, \ldots, \Phi_{n}\right)$ be an $n$-tuple of positive linear mappings $\Phi_{i}: \mathcal{B}(H) \rightarrow \mathcal{B}(K)$ such that $\sum_{i=1}^{n} \Phi_{i}\left(1_{H}\right)=$ $1_{K}$. Let $m_{i}$ and $M_{i}, 0<m_{i} \leq M_{i}$, be the bounds of $A_{i}, i=1, \ldots, n$. For $1 \leq n_{1}<n$, one denotes $m=$ $\min \left\{m_{1}, \ldots, m_{n_{1}}\right\}, M=\max \left\{M_{1}, \ldots, M_{n_{1}}\right\}$, and $\sum_{i=1}^{n_{1}} \Phi_{i}\left(1_{H}\right)=\alpha 1_{K}, \sum_{i=n_{1}+1}^{n} \Phi_{i}\left(1_{H}\right)=\beta 1_{K}$, where $\alpha, \beta>0, \alpha+\beta=1$.

(i) If either $r \leq s, s \geq 1$ or $r \leq s \leq-1$ and also one of two equalities

$$
\mathcal{M}^{[r]}\left(\alpha, \mathbf{A}, \mathbf{\Phi}, 1, n_{1}\right)=\mathcal{M}^{[r]}(1, \mathbf{A}, \mathbf{\Phi}, 1, n)=\mathcal{M}^{[r]}\left(\beta, \mathbf{A}, \mathbf{\Phi}, n_{1}+1, n\right)
$$

is valid, then

$$
\mathcal{M}^{[s]}\left(\alpha, \mathbf{A}, \mathbf{\Phi}, 1, n_{1}\right) \leq \mathcal{M}^{[s]}(1, \mathbf{A}, \mathbf{\Phi}, 1, n) \leq \mathcal{M}^{[s]}\left(\beta, \mathbf{A}, \mathbf{\Phi}, n_{1}+1, n\right)
$$

holds.

(ii) If either $r \leq s, r \leq-1$ or $1 \leq r \leq s$ and also one of two equalities

$$
\mathcal{M}^{[s]}\left(\alpha, \mathbf{A}, \mathbf{\Phi}, 1, n_{1}\right)=\mathcal{M}^{[s]}(1, \mathbf{A}, \mathbf{\Phi}, 1, n)=\mathcal{M}^{[s]}\left(\beta, \mathbf{A}, \mathbf{\Phi}, n_{1}+1, n\right)
$$

is valid, then

$$
\mathcal{M}^{[r]}\left(\alpha, \mathbf{A}, \mathbf{\Phi}, 1, n_{1}\right) \geq \mathcal{M}^{[r]}(1, \mathbf{A}, \mathbf{\Phi}, 1, n) \geq \mathcal{M}^{[r]}\left(\beta, \mathbf{A}, \mathbf{\Phi}, n_{1}+1, n\right)
$$

holds.

Proof. (i) We prove only the case (i). We take $\varphi(t)=t^{r}$ and $\psi(t)=t^{s}$ for $t>0$. Then $\psi \circ \varphi^{-1}(t)=$ $t^{s / r}$ is concave for $r \leq s, s \leq 0$, and $r \neq 0$. Since $-\psi^{-1}(t)=-t^{1 / s}$ is operator monotone for $s \leq-1$ and (3.23) is satisfied, then by applying Theorem 3.1(i') we obtain (3.24) for $r \leq s \leq-1$.

But, $\psi \circ \varphi^{-1}(t)=t^{s / r}$ is convex for $r \leq s, s \geq 0$, and $r \neq 0$. Since $\psi^{-1}(t)=t^{1 / s}$ is operator monotone for $s \geq 1$, then by applying Theorem 3.1(i) we obtain (3.24) for $r \leq s, s \geq 1$, and $r \neq 0$. 
If $r=0$ and $s \geq 1$, we put $\varphi(t)=\ln t$ and $\psi(t)=t^{s}, t>0$. Since $\psi \circ \varphi^{-1}(t)=\exp (s t)$ is convex, then similarly as above we obtain the desired inequality.

In the case (ii) we put $\varphi(t)=t^{s}$ and $\psi(t)=t^{r}$ for $t>0$ and we use the same technique as in the case (i).

\section{References}

[1] B. Mond and J. E. Pečarić, "Converses of Jensen's inequality for several operators," Revue d'Analyse Numérique et de Théorie de l'Approximation, vol. 23, no. 2, pp. 179-183, 1994.

[2] F. Hansen, J. Pečarić, and I. Perić, "Jensen's operator inequality and its converses," Mathematica Scandinavica, vol. 100, no. 1, pp. 61-73, 2007.

[3] J. Mićić, J. Pečarić, and Y. Seo, “Converses of Jensen's operator inequality,” Operators and Matrices, vol. 4, no. 3, pp. 385-403, 2010.

[4] J. Mićić, Z. Pavić, and J. Pečarić, “Jensen's inequality for operators without operator convexity," Linear Algebra and its Applications, vol. 434, no. 5, pp. 1228-1237, 2011. 


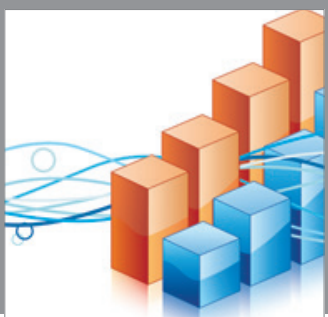

Advances in

Operations Research

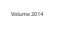

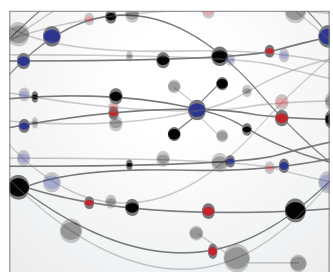

\section{The Scientific} World Journal
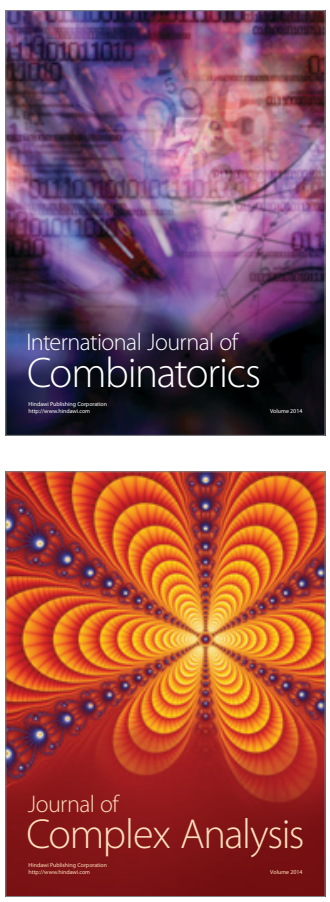

International Journal of

Mathematics and

Mathematical

Sciences
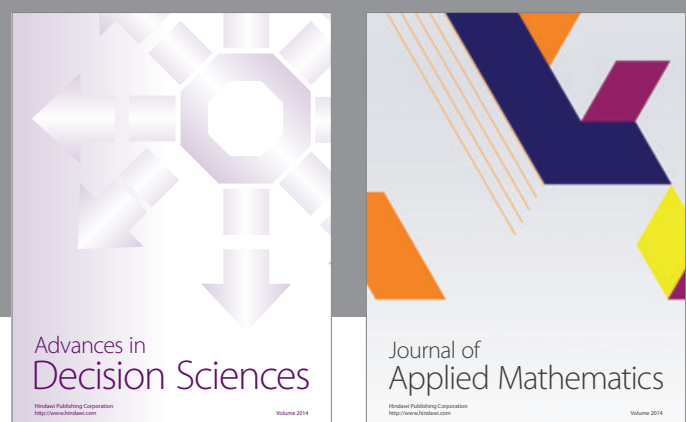

Journal of

Applied Mathematics
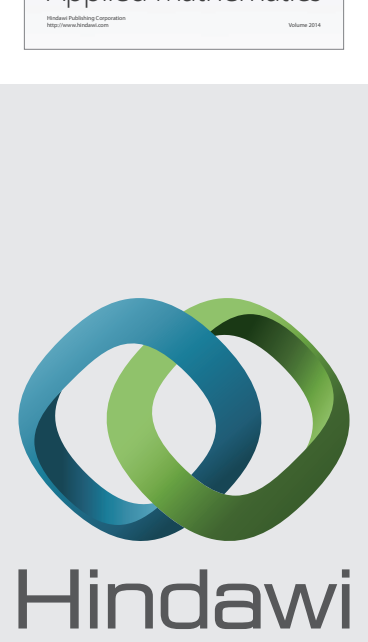

Submit your manuscripts at http://www.hindawi.com
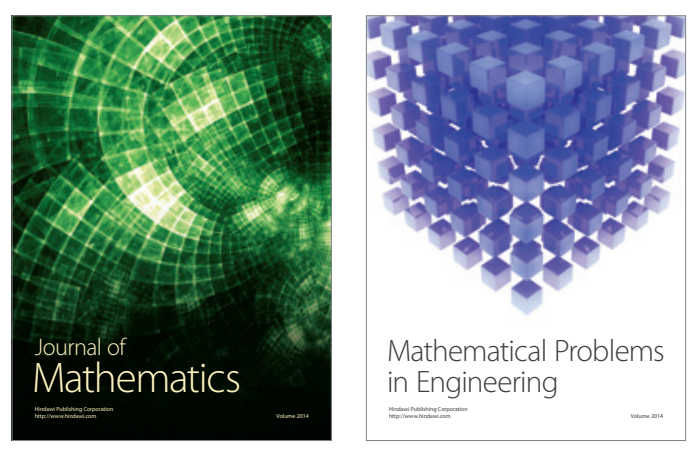

Mathematical Problems in Engineering
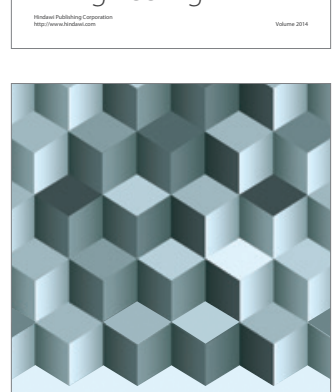

Journal of

Function Spaces
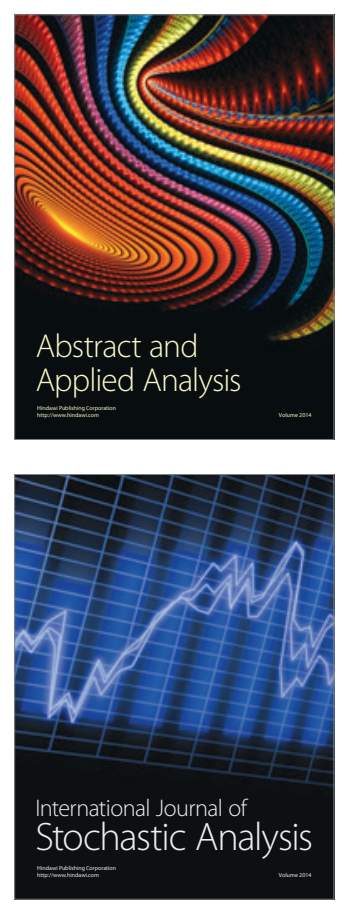

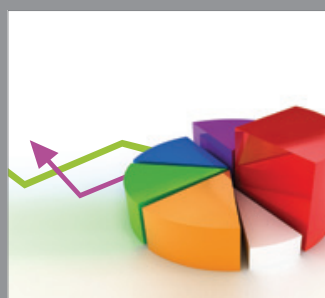

ournal of

Probability and Statistics

Promensencen
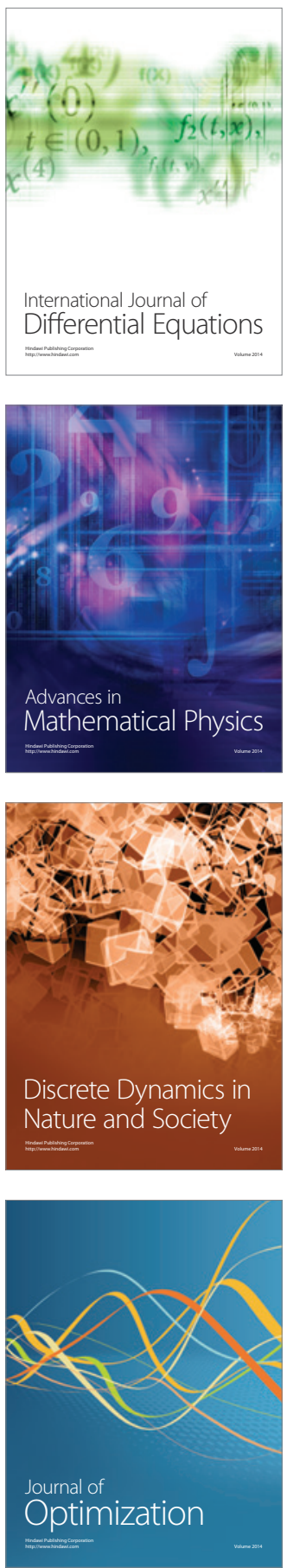